\title{
Determination of Diloxanide Furoate and Metronidazole in Binary Mixture Using First Derivative of the Ratio-Spectra and High-Performance Liquid Chromatography-UV Methods
}

\author{
Nora H. Al-Shaalan \\ Chemistry Department, Girls College of Education in Riyadh, Saudi Arabia
}

\begin{abstract}
Two methods are presented for simultaneous determination of diloxanide furoate and metronidazole. The first method depends on first derivative of the ratio-spectra by measurements of the amplitudes at 242.5 and $285.5 \mathrm{~nm}$ for diloxanide furoate and 225.5 and $300 \mathrm{~nm}$ for metrinidazole. Calibration graphs were established for $2-100 \mu \mathrm{g} \mathrm{mL}^{-1}$ for diloxanide furoate and $1-50 \mu \mathrm{g} \mathrm{mL}^{-1}$ for metronidazole. In the second method (HPLC), a reversed-phase column with a mobile phase of ethylacetate:chloroform:dioxin:methanol $(8: 7: 2: 3(\mathrm{v} / \mathrm{v} / \mathrm{v} / \mathrm{v}))$ at $0.9 \mathrm{~mL} \mathrm{~min}{ }^{-1}$ flow rate was used to separate both compounds with detection at $250-345 \mathrm{~nm}$. Linearity was obtained in the concentration range of $0.025-50 \mu \mathrm{g} \mathrm{mL}^{-1}$ for diloxanide furoate and $0.15-50 \mu \mathrm{g} \mathrm{mL}^{-1}$ for metronidazole. All of the proposed methods have been extensively validated. These methods allow a number of cost and time saving benefits. The described methods can be readily utilized for analysis of pharmaceutical formulations. There was no significant difference between the performance of the proposed methods regarding the mean values and standard deviations. The described HPLC method showed to be appropriate for simultaneous determination of diloxanide furoate and metronidazole in pharmaceutical dosage forms and in hyman serum samples.
\end{abstract}

Key words: HPLC, diloxanide furoate, metronidazole, chemometric spectrophotometry, pharmaceutias, human serum

\section{INTRODUCTION}

Diloxanide furoate is the drug of choice in the treatment of asymptomatic intestinal amoebiasis ${ }^{[1]}$. The most recent methods for determination of diloxanide furoate included chromatographic ${ }^{[2-5]}$, electrochemical ${ }^{[6-}$ ${ }^{9]}$ and spectrophotometric ${ }^{[10-13]}$ techniques.

Mertonidazole has strong antiprotozoal and bactericidal actions ${ }^{[1]}$. Several methods have been reported for metrronidazole assay including chromatographic $^{[14-16]}$, electrochemical ${ }^{[17-19]}$ and spectrophotometric ${ }^{[20-23]}$ techniques.<smiles>C=C(C(C)C)I(I)c1ccc(C)cc1</smiles>

Diloxanide

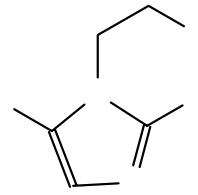

Metronidazole compounds with overlapping spectra $^{[13-15]}$. The principles and advantages of this technique have been described by O'Haver and Green ${ }^{[24]}$.

HPLC methods are useful in the determination of drugs in pharmaceutical dosage forms and biological sample. Owing to the widespread use of HPLC in routine analysis, it is important that good HPLC methods are developed and that these are thoroughly validated $^{[24-26]}$.

The purpose of the present study was to investigate the utility of derivative ratio spectrophotometry and HPLC in the assay of diloxanide furoate and metronidazole, in combination, in the pharmaceutical preparations without the necessity of sample pretreatment. We also performed a study concerning simultaneous determination of these compounds in human serum samples using HPLC technique. Two methods were developed and validated in this work: a first derivative spectrophotometric of the ratio-spectra and gradient HPLC methods for the simultaneous determination of diloxanide furoate and metronidazole. The methods had sufficiently good accuracy, precision and permitted a simple, time-and money- saving assay of these compounds in mixtures.

\section{MATERIALS AND METHODS}

Derivative spectrophotometry is an analytical technique of great utility for resolving some mixtures of
Apparatus and conditions: The Hewlett-Packard liquid chromatographic system consisted of a gradiant

Corresponding Author: $\quad$ Nora H. Al-Shaalan, Chemistry Department, Girls College of Education in Riyadh; Saudi Arabia 
Quat pump Model G 1311 A (HP, Avondale, PA, USA) connected with a HP G 1314 A UV-VIS detector (HP, Avondale, PA, USA) operating at $265 \mathrm{~nm}$, a G $1328 \mathrm{~A}$ (Cotati, CA) injection valve, with a $20 \mu \mathrm{L}$ loop. The chromatographic data were collected and analyzed using HP Chem Station for LC and LC/MS system (Hewlett-Packard, Avondale, PA, USA). The chromatographic separation was performed at ambient temperature (20-22 oC) using an analytical column, Spherisorb $\AA$, $5 \mu \mathrm{m}, 4.6$ × $150 \mathrm{~mm}$ i.d., (Waters, Milford, MA, USA).

The reversed mobile phase was obtained by mixing ethylacetate:chloroform:dioxin:methanol $\quad$ (8:7:2:3 $(\mathrm{v} / \mathrm{v} / \mathrm{v} / \mathrm{v}))$. The flow rate was $0.9 \mathrm{~mL} \mathrm{~min}^{-1}$. Finasteride was used as an internal standard. An amount of $20 \mu \mathrm{L}$ of each solution was injected and chromatograms were recorded.

Spectrophotometric measurements were carried out on a computerized Spectronic Gensys 2PC, UV/visible Spectrophotometer (Milton Roy, USA), using $1.00 \mathrm{~cm}$ quartz cells. The obtained spectral data were saved in PC apparatus program and the subsequent statistical manipulation was performed by transferring the spectral data to Microsoft excel XP program and processing them with the standard curve fit package and matrix calculations. Sonicator (Bransonic 220 / BenderHobein), Heater(Salvis) (Heidolph). Balance (Mettler Toledo). Curve Expert version 1.37 Copyright $^{\circledR} 1995$ 2001 by Daniel Hyams. GraphPad Instat version $3.05,32$ bit for win 95/NT created Sep. 27,2000 Copyright $^{\mathcal{O}} 1992-2000$ by GraphPad software.

Chemicals and reagents: All chemicals were of analytical-reagent grade and solvents were of spectrophotometric and HPLC grade.

Diloxanide furoate and metronidazole and its pharmaceytical dosage form were kindly provided by the Rhone Poulenc Co., Paris, finasteride used, as internal standard was supplied from Aldrich.

HPLC methanol, ethyl acetate, chloroform and dioxane (Merk, Darmstadt, Germany). For spectrophotometric studies, methanol was purchased from Merk (Darmstafd, Germany).

Preparation of standard solutions and calibration: Stock standard solutions of each of diloxanide furoate and metronidazole were prepared separately by dissolving $10 \mathrm{mg}$ of each drug in $10 \mathrm{~mL}$ methanol.

The standard solutions were prepared individually by dilution of the stock solutions with methanol for spectrophotometric experiments and with mobile phase for chromatographic experiments to reach concentration range of 1-50 $\mu \mathrm{g} \mathrm{mL}^{-1}$ for diloxanide furoate and 2-100 $\mu \mathrm{g} \mathrm{mL} L^{-1}$ for metronidazole and $0.025-50 \mu \mathrm{g} \mathrm{mL}^{-1}$ for diloxanide furoate and $0.15-50 \mu \mathrm{g} \mathrm{mL^{-1 }}$ for metronidazole, respectively.

\section{Procedures}

For first derivative of the ratio spectrophotometric method (DD1): According to the theory of the ratiospectra derivative method ${ }^{[27,28]}$. For diloxanide furoate; the stored UV absorption spectra of standard solutions of diloxanide furoate were divided, wavelength-bywavelength, by a standard spectrum of metronidazole $\left(20 \mu \mathrm{g} \mathrm{mL}^{-1}\right)$. The first derivative was calculated for the obtained spectra with $\Delta \lambda=4 \mathrm{~nm}$. The amplitudes at 245.5 and $251.3 \mathrm{~nm}$ were measured and found to be in linear relation to the concentration of diloxanide furoate.

For metronidazole, the stored UV absorption spectra of standard solutions of metronidazole were divided, wavelength-by-wavelength, by a standard spectrum of diloxanide furoate $\left(15 \mu \mathrm{g} \mathrm{mL}{ }^{-1}\right)$. The first derivative was calculated for the obtained spectra with $\Delta \lambda=4 \mathrm{~nm}$. The amplitudes at 235.3 and $266.4 \mathrm{~nm}$ were measured and found to be in linear relation to the concentration of metronidazole.

For high-performance liquid chromatographic method: Standard solutins were prepared separately with mobile phase by varying concentrations of diloxanide furoate and metronidazole in the range $0.025-50$ and $0.15-50 \mu \mathrm{g} \mathrm{mL}^{-1}$, respectively. Maintaining concentration of finasteride (IS) at a constant level of $25 \mu \mathrm{g} \mathrm{mL}^{-1}$.

Trplpicate $10 \mu \mathrm{L}$ injections were made for each solution and peak area ratio of each concentration to the internal standard was plotted against the corresponding concentration to obtain the calibration graph.

All of the proposed methods were validated as to precision (reported as the relative standard deviation, R.S.D. (\%)), linearity (evaluated by regression equations), detection and determination limits and accuracy. The limit of detection (LOD) and limit of quantitation (LOQ) of the procedures are also shown in Table 1, which were calculated according to the 3 and $10 \mathrm{~s} \mathrm{~m}^{-1}$ criterions, respectively, where $\mathrm{s}$, is the standard deviation of the absorbance $(n=4)$ of the sample and $\mathrm{m}$ is the slope of the corresponding calibration curve.

The ruggedness and precision were checked in the same and different Days. The R.S.D. (\%) was calculated to check the ruggedness and precision of the methods. Accuracy was determined by recovery studies.

For tablets: For all methods, 10 tablets (labeled to contain $250 \mathrm{mg}$ of diloxanide furoate and $200 \mathrm{mg}$ of metronidazole and excipients) were weighed and finely powdered. An accurate weight of the powder equivalent to one tablet content was accurately weighed, transferred into a $100 \mathrm{~mL}$ calibrated flask, diluted with methanol, stirred for about $10 \mathrm{~min}$ and then completed to volume with the same solution. This solution was filtered to separate any insoluble matter. The filtrate 
was collected in a clean flask. Appropriate solutions were prepared by taking suitable aliquots of clear filtrate (adding the constant amount of internal standard for HPLC study) and diluting with selected solvent in order to obtain a final solution. The assay was completed as described under section 2.4.1 and 2.4.2. The amounts of diloxanide furoate and metronidazole were calculated from the corresponding regression equations.

Percent recovery study: To study the accuracy of the proposed methods and to check the interference from excipients used in the dosage forms, recovery experiments were carried out by applying the standard addition technique.

This study was performed by adding known amounts of diloxanide furoate and metronidazole to a known concentration of the commercial tablets. The resulting mixtures were analyzed as described earlier.

Recovery studies in human serum by HPLC: Serum sample, obtained from healthy individuals (after obtaining their written consent), were stored frozen until assay. After gentle, thawing aliquots of serum taken in a graduated tube were spiked with diloxanide furoate and metronidazole (dissolved in methanol) to achieve a final concentration of 500 and $1000 \mu \mathrm{g} \mathrm{mL}^{-1}$, respectively then treated with $500 \mu \mathrm{L}$ of acetonitrile as serum protein precipitating agent, the volume was completed to $2 \mathrm{~mL}$ with the same serum sample. The tubes were vortexed for $5 \mathrm{~min}$ at $1500 \mathrm{~g}$ and then centrifuged for $10 \mathrm{~min}$ at $5000 \mathrm{x}$ g for getting ride of protein residues. The supernatant was taken carefully, serum samples including various concentrations of diloxanide furoate and metronidazole and constant amount of internal standard were injected into the column.

\section{RESULTS AND DISCUSSION}

DD1 method: As shown in Fig. 1, it can be seen that the zero order absorption spectra of diloxanide furoate and metronidazole are very overlapped and as a result, the simultaneous determination of the two drugs cannot be possible for reliable direct absorbance measurements.

To optimize the simultaneous determination of diloxanide furoate and metronidazole by using DD1 method, it is necessary to test the influence of the variables: divisor standard concentration, $\Delta \lambda$ and smoothing function. All these variables were studied. This influence of $\Delta \lambda$ for obtaining the first derivative of the ratio-spectra was tested and $\Delta \lambda=4 \mathrm{~nm}$ was selected. An accurate choice of divisor standard concentration is fundamental for several reasons ${ }^{[25,26]}$, hence, we tested the methods with various divisor concentrations. The results of all the tests are not shown for the sake of brevity and because these do not add to

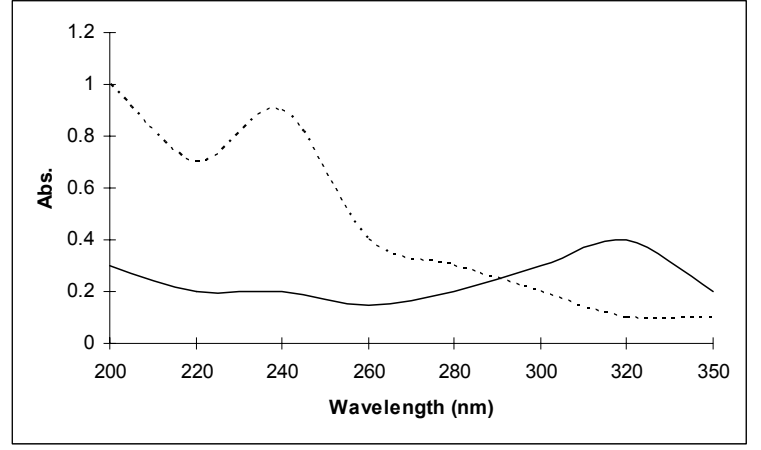

Fig. 1: Absorption spectra of diloxanide furoate $(-)$, $30 \mu \mathrm{g} \mathrm{mL}^{-1}$ and metronidazole (‥), $30 \mu \mathrm{g} \mathrm{mL}^{-1}$

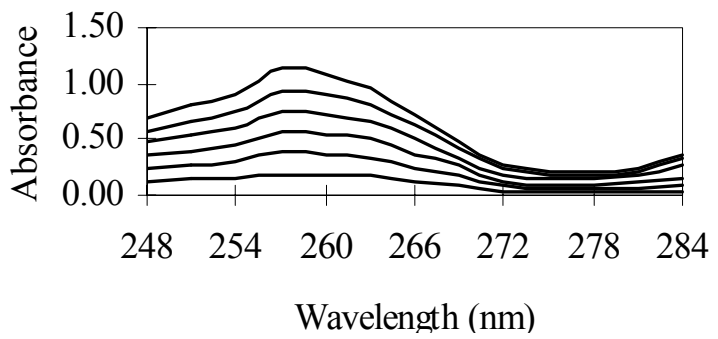

Fig. 2: Ratio spectra of diloxanide furoate (1-50 $\mu \mathrm{g}$ $\left.\mathrm{mL}^{-1}\right)$. Divisor is $20.4 \mu \mathrm{g} \mathrm{mL}^{-1}$ metronidazole

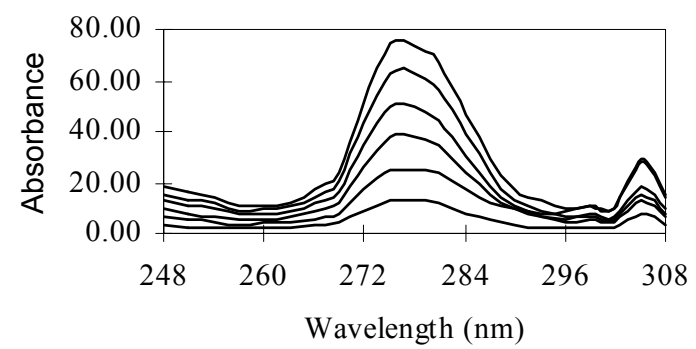

Fig. 3: Ratio spectra of metronidazole (2-100 $\mu \mathrm{g}$ $\mathrm{mL}^{-1}$ ). Divisor is $50.70 \mu \mathrm{g} \mathrm{mL} \mathrm{m}^{-1}$ diloxanide fyroate

the scientific value of the work. A standard spectrum of $20 \mu \mathrm{g} \mathrm{mL}^{-1}$ of diloxanide furoate was considered as suitable for the metronidazole determination.

In Fig. 2 and 3, two series of the ratio-spectra of diloxanide furoate/metronidazole and metronidazole/ diloxanide furoate were shown. The corresponding first derivative of the ratio-spectra of Fig. 4 and 5 were shown. For calibration graphs, the wavelengths were selected which exhibited the best linear response to the analyte concentration, i.e. in the first derivative mode 239.6 and $255.1 \mathrm{~nm}$ for diloxanide furoate and 233.4 and $266.5 \mathrm{~nm}$ for metronidazole. The calibration graphs of each drug at both wavelengths were achieved by plotting the values of the first derivative of the ratiospectra diloxanide furoate/metronidazole and metronidazole/diloxanide furoate, with variable concentrations of diloxanide furoate and metronidazole. The proposed method is applicable over the ranges 1-50 $\mu \mathrm{g} \mathrm{mL} L^{-1}$ for diloxanide 
Table 1: Statistical data for the calibration graphs of diloxanide furoate and metronidazole by DD1 and HPLC methods

\begin{tabular}{|c|c|c|c|c|c|c|}
\hline & \multicolumn{3}{|c|}{ Diloxanide } & \multicolumn{3}{|c|}{ Metronidazole } \\
\hline & DD1 & & HPLC & DD1 & & HPLC \\
\hline Linearity range $\left(\mu \mathrm{g} \mathrm{mL}^{-1}\right)$ & $1-50$ & $1-50$ & $0.025-50$ & $2-100$ & $2-100$ & $0.15-50$ \\
\hline Slope & 0.021 & 0.005 & 0.156 & 0.003 & 0.012 & 0.190 \\
\hline Intercept & -0.003 & 0.001 & 0.033 & 0.005 & 0.002 & 0.008 \\
\hline Correlation coefficient & 0.9997 & 0.9993 & 0.9999 & 0.9992 & 0.9994 & 0.9999 \\
\hline R.S.D. of the slope & 0.25 & 0.53 & 0.17 & 0.33 & 0.62 & 0.70 \\
\hline R.S.D. of the intercept & 0.06 & 0.17 & 0.55 & 0.05 & 0.19 & 0.71 \\
\hline $\operatorname{LOD}\left(\mu \mathrm{g} \mathrm{mL}^{-1}\right)$ & 0.04 & 0.04 & 0.005 & 0.02 & 0.11 & 0.006 \\
\hline $\operatorname{LOQ}\left(\mu \mathrm{g} \mathrm{mL}^{-1}\right)$ & 0.66 & 0.17 & 0.020 & 0.11 & 0.45 & 0.039 \\
\hline Repeatability (R.S.D.; \%) & 0.53 & 0.70 & 0.80 & 0.39 & 0.34 & 0.95 \\
\hline Reproducibility (R.S.D.; \%) & 0.81 & 0.99 & 0.90 & 0.56 & 0.46 & 0.48 \\
\hline
\end{tabular}

Table 2: Determination of dioxanide furoate and metronidazole in authentic, laboratory prepared mixtures and pharmaceutical dosage form using first derivative of the ratio-spectra (DD1), HPLC and reported methods

\begin{tabular}{|c|c|c|c|c|}
\hline Standard solution & Reported & HPLC & DD1 & DD1 \\
\hline \multirow[t]{3}{*}{ Authentic diloxanide } & $99.9 \pm 0.44$ & $99.8 \pm 0.42$ & $100.1 \pm 0.48$ & $99.0 \pm 0.40$ \\
\hline & & $\mathrm{t}=0.65$ & $\mathrm{t}=0.69$ & $\mathrm{t}=0.88$ \\
\hline & & $F=1.09$ & $\mathrm{~F}=1.21$ & $\mathrm{~F}=1.19$ \\
\hline \multirow[t]{3}{*}{ Authentic metronidazole } & $100.3 \pm 0.52$ & $99.9 \pm 0.52$ & $100.1 \pm 0.55$ & $99.8 \pm 0.51$ \\
\hline & & $\mathrm{t}=0.66$ & $\mathrm{t}=0.60$ & $\mathrm{t}=1.55$ \\
\hline & & $\mathrm{F}=1.00$ & $\mathrm{~F}=1.11$ & $\mathrm{~F}=1.17$ \\
\hline \multirow{3}{*}{$\begin{array}{l}\text { Laboratory prepaed mix. } \\
\text { for diloxanide }\end{array}$} & $100.1 \pm 0.71$ & $100 \pm 0.67$ & $100.1 \pm 0.75$ & $99.9 \pm 0.70$ \\
\hline & & $\mathrm{t}=0.24$ & $\mathrm{t}=0.24$ & $\mathrm{t}=0.50$ \\
\hline & & $\mathrm{F}=1.11$ & $\mathrm{~F}=1.12$ & $\mathrm{~F}=1.02$ \\
\hline \multirow{3}{*}{$\begin{array}{l}\text { Laboratory prepared mix. } \\
\text { for metronidazole }\end{array}$} & $99.9 \pm 0.72$ & $100.1 \pm 0.71$ & $100.2 \pm 0.72$ & $99.8 \pm 0.73$ \\
\hline & & $\mathrm{t}=0.42$ & $t=0.68$ & $\mathrm{t}=0.21$ \\
\hline & & $\mathrm{F}=1.01$ & $F=1.00$ & $\mathrm{~F}=1.01$ \\
\hline \multirow{3}{*}{$\begin{array}{l}\text { Furamebe forte tab. } \\
\text { for diloxanide furoate }\end{array}$} & $100.4 \pm 0.62$ & $99.9 \pm 0.64$ & $100.3 \pm 0.59$ & $99.8 \pm 0.57$ \\
\hline & & $\mathrm{t}=0.33$ & $\mathrm{t}=0.29$ & $\mathrm{t}=0.88$ \\
\hline & & $\mathrm{F}=1.02$ & $\mathrm{~F}=1.08$ & $\mathrm{~F}=1.02$ \\
\hline \multirow{3}{*}{$\begin{array}{l}\text { Furamebe forte tab. } \\
\text { for metronidazole }\end{array}$} & $100.0 \pm 0.59$ & $100.1 \pm 0.58$ & $100.2 \pm 0.60$ & $99.9 \pm 0.55$ \\
\hline & & $\mathrm{t}=0,60$ & $\mathrm{t}=0.57$ & $\mathrm{t}=0.92$ \\
\hline & & $\mathrm{F}=1.01$ & $F=1.02$ & $\mathrm{~F}=1.20$ \\
\hline \multicolumn{3}{|c|}{ Standard addition technique for diloxanide furoate } & $99.6 \pm 0.70$ & $99.5 \pm 0.73$ \\
\hline \multicolumn{3}{|c|}{ Standard addition technique for metronidazole } & $100.3 \pm 0.67$ & $99.6 \pm 0.70$ \\
\hline
\end{tabular}

Theoretical values at $95 \%$ confidence limit are

$\mathrm{t}=2.306$ and $\mathrm{F}=6.39\left(\mathrm{n}_{1}=5, \mathrm{n}_{2}=5\right)$

Table 3: Results obtained for dioxanide furoate and metronidazole analysis from human serum using HPLC method

\begin{tabular}{lcccc}
\hline & \multicolumn{3}{c}{ Diloxanide } & \multicolumn{3}{c}{ Metronidazole } \\
&.---------- & ---- \\
Added $\left(\mu \mathrm{g} \mathrm{mL}^{-1}\right)$ & 2.00 & 10.00 & 10.00 & 30.00 \\
\hline $\mathrm{n}$ & 4 & 4 & 4 & 4 \\
Found $\left(\mu \mathrm{g} \mathrm{mL}^{-1}\right)$ & 1.98 & 9.81 & 9.78 & 29.38 \\
R.S.D. $(\%)$ & 1.04 & 0.7 & 0.84 & 0.94 \\
Average recovery (\%) & 99.20 & 98.07 & 97.80 & 97.93 \\
\hline
\end{tabular}

furoate and 2-100 $\mu \mathrm{g} \mathrm{mL} \mathrm{m}^{-1}$ for metronidazole. The characteristic parameters, necessary statistical data of the regression equations, LOD and LOQ values and reproducibility data are complied in Table 1 .

Repeatability and reproducibility variables were characterized by R.S.D.(\%) and by the difference between theoretical and measured concentrations. There was no significant difference for the assay, which was tested within-day (repeatability) and between-days (reproducibility).

In order to demonstrate the validity and applicability of the proposed DD1 method, recovery studies were performed by analyzing synthetic mixtures of diloxanide furoate and metronidazole, with different composition ratios (Table 2).

High-performance liquid chromatographic method: Drug analysis is undertaken during various phases of pharmaceutical development, such as formulation and stability studies, quality control and pharmacological testing in animals and humans. All these investigations require reliable and validated analytical methods in order to measure drugs in pharmaceutical formulations and biological samples.

In order to effect the simultaneous elution of diloxanide furoate and metronidazole peaks under gradient conditions, the mixtures of ethylacetate, chloroform, dioxin and methanol in different combinations at various flow rates were assayed. The optimum wavelength for detection was $265 \mathrm{~nm}$ at which much better detector responses for both drugs were obtained. The mixture of ethylacetate:chloroform:dioxin:methanol (8:7:2:3 $(\mathrm{v} / \mathrm{v} / \mathrm{v} / \mathrm{v}))$ at $0.9 \mathrm{~mL} \mathrm{~min}^{-1}$ flow rate, proved to be better than the other mixtures and flow rates for separation, 


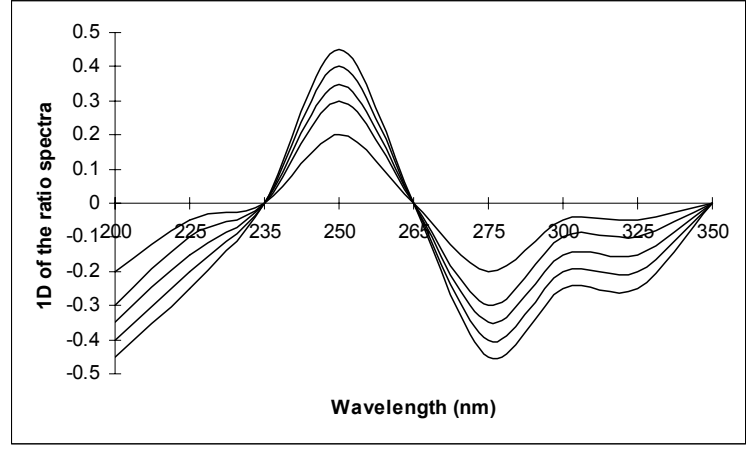

Fig. 4: First derivative of the ratio-spectra of different concentration of diloxanide furoate and divisor metronidazole

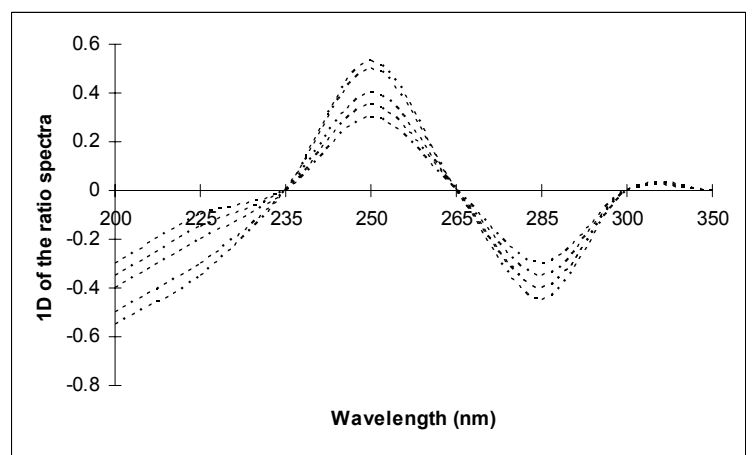

Fig. 5: First derivative of the ratio-spectra of different concentration of metronidazole and divisor diloxanide furoate

since the chromatographic peaks were better defined, resolved and free from tailing. The retention times were $2.06 \mathrm{~min}$ for diloxanide furoate, $3.36 \mathrm{~min}$ for metronidazole and 4.32 min for finasteride (IS).

In HPLC methods, precision and accuracy can often be enhanced by the use of an appropriate internal standard, which also serves to correct for fluctuations in the detector response. Ideally, an internal standard should display similar physico-chemical properties to the analytes. But it did not obtained good resolution and peak shape with the similar compounds. The structure of finasteride is not similar to dilxanide furoate and metronidazole. However, it was chosen as the internal standard because it showed a shorter retention time with better peak shapes and better resolution, compared to other potential internal standards.

System suitability tests are an integral part of a liquid chromatographic method. System suitability tests are used to verify that the resolution and reproducibility of the chromatographic system are adequate for the analysis to be done. System suitability tests were carried out according to USP 24, method $(621)^{[27]}$ on the chromatogram of freshly prepared standard solutions to check various parameters, such as resolution, selectivity, tailing and capacity factors. Resolution and selectivity factors for this system were found 2.17 and 2.48, respectively. The tailing and capacity factors were obtained as 1.11 and 0.75 for diloxanide furoate and 1.33 and 1.85 for metronidazole, respectively. The variation in retention times among six replicate injections of diloxanide furoate and metronidazole standard solutions was very low, rendering a R.S.D of 0.98 and $0.62 \%$, respectively. The results obtained from system suitability tests are in agreement with the USP requirements.

Peak area ratios (Asample\AIS) were plotted against corresponding concentrations in the range of 0.025-50 $\mu \mathrm{g} \mathrm{mL}^{-1}$ for diloxanide furoate and $0.15-50$ $\mu \mathrm{g} \mathrm{mL}^{-1}$ for metronidazole. Linear regression parameters of the peak area rations versus concentrations of diloxanide furoate and metronidazole are presented in Table 1 . The results showed highly reproducible calibration curves with correlation coefficients of $>0.999$. Necessary statistical data of the regression equations, such as LOD value, LOQ value, repeatability and reproducibility data were also as shown in Table 1. Repeatability and reproducibility variabilities were characterized by R.S.D. (\%) (Table 1). According to these results, there was no significant difference for the assay.

In order to demonstrate the validity and applicability of the proposed HPLC method, recovery tests were carried out by analyzing in the synthetic mixtures of diloxanide furoate and metronidazole, in different composition ratios (Table 3 ).

Analysis of tablets: When working on synthetic mixtures, results encourage the use of the proposed methods described here for the assay of diloxanide furoate and metronidazole in commercial tablet dosage forms. The two proposed methods can be used for the simultaneous determination of diloxanide furoate and metronidazole in the presence of each other and without prior separation of the excipients. Each film-coated tablet contain $250 \mathrm{mg}$ of diloxanide furoate, $200 \mathrm{mg}$ of metronidazole and the inactive ingredients colloidal silicon dioxide, magnesium stearate, microcrystalline cellulose and sodium starch glycolate. The substances were eluted, forming well shaped, symmetrical single peaks, well separated from the solvent front. No interfering peaks were obtained in the chromatogram due to tablet excipients. The utility of all of the proposed methods was verified by means of replicate estimations of pharmaceutical preparations and results obtained were evaluated statistically. The results obtained for the analysis of tablets by spectrophotometric and HPLC methods (Table 2) indicate that it can be used for routine quality control analysis of this binary mixture in pharmaceuticals.

A comparison with an official or reference method has not been possible in any pharmacopoeias or literatures, because so far no other procedure for the quantitation of diloxanide furoate and metronidazole from pharmaceutical formulations has been reported. The proposed spectrophotometric and HPLC results 
were compared with reported method ${ }^{[28]}$. According to the student's t-test and variance ratio F-test, the calculated $\mathrm{t}$-and $\mathrm{F}$-values were less than the tabulated values at $95 \%$ confidence level, indicating no significant difference between the performance of all the proposed and reported methods as regards to mean values and standard deviations (Table 2).

Recovery studies were realized for the tablets regarding accuracy and precision of the proposed methods applying standard addition technique. The results in Table 2 concluded that the proposed methods are sufficiently accurate and precise in order to be applied for pharmaceutical dosage forms. High percentage recovery data show that all of the proposed methods are free from interference of excipients used in the formulations.

Application to the serum samples: In order to check the applicability of the proposed HPLC method to biological materials, the recovery studies were performed in human serum samples. Analysis of drugs from serum by HPLC usually requires extensive timeconsuming sample preparation, use of expensive organic solvent and other chemicals ${ }^{[29,30]}$. In our proposed method, the serum protein is precipitated by addition of acetonitrile, centrifugation on $5000 \mathrm{~g}$ and the supernatant is diluted, directly injected and analyzed.

The typical chromatogram obtained from the serum spiked with diloxanide furoate, metronidazole and IS (a) and blank serum (b), indicates no interference from the endogenous substances present in serum. Serum samples were spiked with two different concentrations of diloxanide furoate and metronidazole and constant level of IS. The determined results and recoveries (Table 3) indicate that the proposed method gives reproducible results, is easy to perform and is sensitive enough.

\section{CONCLUSION}

The DD1 and HPLC methods enable the quantitation of mixtures of diloxanide furoate and metronidazole with good accuracy and precision, either in laboratory made mixtures or in pharmaceutical dosage forms.

Comparison of the results obtained with the three methods, indicate no substantial difference. A little superiority of the HPLC method over the spectrophotometric techniques is indicated from the limits of detection and determination and correlation coefficients (Table 1).

All procedures are rapid, precise and work without solving equations or separation steps. The DD1 method has great sensitivity and accuracy.

\section{REFERENCES}

1. El-Hawary, M.B., M.T. khayyall and Z. Isaak, 1985. Hand Book of Pharmacology. The Scientific Book Center, S.O.P. Presee, Cairo.

2. Indravadan, C.B., B. Raman, M. Sundaresan and M.B. Ashok, 1997. High Performance Thin Layer Chromatographic Analysis of Certain Drugs. Anal. Chim. Acta, 354: 123-128.

3. Prasad, C.V., V. Sripriya, R.N. Saha and P. Parimoo, 1999. Chromatographic analysis of certain binary mixtures. J. Pharm. Biomed. Anal., 21: 961-8.

4. Hasan, N.Y., M.A. Elkawy, B.E. Elzeany and N.E. Wagieh, 2002.Utility of chromatographic technique for analysis of certain drugs. J. Pharm. Biomed. Anal., 28: 187-197.

5. Ishal, A. and D. Sober, 2005. Gas chromatographic analysis of certain furoates J. Pharm. Biomed. Anal., 39: 819-23.

6. Parthasarathy, P.C., L. Ananthan, M.A. Likhate, S.G. Manjunatha and N. Kalyanam, 1993. Potentiometric analysis of certain drugs in presence of their degradated products. E. J. Med. Chem., 28: 195-199.

7. Jelinek, T., M. Ziegler and T. Loscher, 1994. Usage of polarographic technique in analysis of certain drugs. Dtsch. Med. Wochenschr., 119: 1618-22.

8. Venugopalan, B., B. Patel, P.J. Karnik, N.J. de Souza, D.K. Chatterjee and N. Iyer, 1996. Voltametric analysis of diloxaanide furoate. E. J. Med. Chem., 31: 485-488.

9. Rosane, M., N. Maria, M. Jacir, V. José and Y. Rosendo, 2004. Electrochemical analysis of cerain pharmaceutical compounds. J. Inc. Pheno., 50: 151-155.

10. Al-Ghanam, S.M. and F. Belal, 2001. Spectrophotometric analysis of certain drugs in presence of their metabolites. Il Farmaco, 56: 677681.

11. Galal, S.M., M.M. Bedair and M.A. el-Sayed, 1991. Spectrophotometric determination of two pharmaceutical dosage forms in presence of each other. J. Pharm. Belg., 46: 315-9.

12. Sethi, P.D., P.K. Chatterjee and C. Jain, 1988. Colorimetric analysis of diloxanide furoate. J. Pharm. Biomed. Anal., 6: 253-258.

13. Talwar, S.K., S.C. Sharma and S. Das, 1986.Charge transfer reactions for determinationof diloxanide furoate. J. Pharm. Biomed. Anal., 4: 511-515.

14. Frasey, A.-M., B.-G. Anne, M-P. Pouget, M. Vennat, B. Lartigue, C. Galmier, M.-J. Galmier, 2004. Chromatographic analysis of metronidazole. Micro. Acta, 144: 171-176. 
15. Akay, C., S.A. Ozkan, Z. Senturk and S. Cevheroglu, 2003. Liquid chromatographic analysis of certain antimicrobials. Il Farmaco, 57: 953-957.

16. Rizk, M., F. Belal, F. Ibrahim, S. Ahmed and Z.A. Sheribah, 2002. Detection of metronidazole in pharmaceutical dosage forms. J. Pharmaceut. Biomed. Anal., 27: 813-820.

17. Bollo, S., L.J. Nunez-Vergara, J.A. Squella, 2004. Voltametric determination of certain antiaembic drugs. J. Elec. Chem., 562: 9-14.

18. Loke, M.-L, J. Tjornelund and B. Halling-2004. Potentiometric analysis of metronidazole. Sorensen, Chemosphere, 48,: 351-361.

19. Ellenrieder, V., W. Boeck, C. Richter, R. Marre, G. Adler and B. Glasbrenner, 1999. Polarographic analysis of certain pharmaceutical dosage forms. Scan. J. Gas., 34: 750-756.

20. Nagaraja, P., K.R. Sunitha, R.A. Vasantha and H.S. Yathirajan, 2002. Derivative spectrophotometric analysis of certain drugs in presene of their degraded products. J. Pharm. Biomed. Anal., 28: 527-535.

21. Parimoo. P., C.V.N. Prasad and A. Gautam, 1996. Colorimetric analysis of metronidazole in tablets. Inter. J. Pharm., 134: 231-234.

22. Parimoo, P., C.V.N. Prasad and R. Vineeth, 1996. Detection and determination of metronidazole in dosage forms. J. Pharm. Biomed. Anal., 14: 389393.
23. Chen, H., Z. Gong and Z. Zhang, 2006. Chemometric analysis of some binary mixtures. J. Pharm. Biomed. Anal., 41: 1412-1417.

24. O' Haver, T.C. and G.L. Green, 1976. The principles and advantages of derivative spectrophotometric technique. Anal. Chem. 48: 312-318.

25. Ozkan, S.A., C. Akay, S. Cevheroglu and Z. Senturk, 2001. Utility of certain methods for analysis of certain drugs. J. Liq. Chromatogr. Rel. Technol., 24: 983.

26. El-Gindy, A., B. El-Zeany, T. Awand and M.M., Shabana, 2001. Analytical study of some drugs in biological fluids. J. Pharm. Biomed. Anal., 26: 203.

27. The US Pharmacopeia, Easton, Rand Mc Nally Taunton, MA, 2000, $24^{\text {th }}$ Review (CD-ROM).

28. Salem, H., 1995. Derivative spectrophotometric determination of some drugs binary mixture. Zag. J. Pharm. Sci., 4: 47-54.

29. Moor, J.D., G. Valette, A. Darque, X.J. Zho and J.P. Sommadossi, 2002. Analysis of drugs in biological fluids. J. Am. Soc. Mass Spectrom., 11: 1134.

30. Kenney, K.B., S.A. Wring, R.M. Carr, G.N. Well and J.A. Dunn, 2000. Utility of certain analytical techniques for detection of drugs in biological fluids. J. Pharm. Biomed. Anal., 22: 967. 